\title{
Pratiques Festives Des Brouteurs En Côte d'Ivoire Entre Ritualisation De La Fête Et Categorisation Sociale Du Cybercriminel
}

\author{
Bamba Ladji \\ Raymond Nébi Bazare \\ Sociocriminologue, Maître assistant \\ UFR Criminologie — Université Félix Houphouet Boigny \\ doi: 10.19044/esj.2016.v12n17p198 URL:http://dx.doi.org/10.19044/esj.2016.v12n17p198
}

\begin{abstract}
This article aims to explain the social categorization mechanism that governs the world of cybercriminals. It is therefore a practical review of the "grazer" including festive practices (sex, alcohol, psychotropic, etc.) that the ethnographic approach allowed to do. The analysis of both qualitative and quantitative data, put light on the complexity of the social processes of the Ivorian typologisation cybercriminal and festive practices related thereto. Thus the daily "bulldozer" and the ritualistic nature of existing social practices were observed over one year (February 2012 - January 2013), among 250 respondents of which 100 criminals on all 10 districts of Abidjan. This allowed to go to the evidence that festive practices are the ends pursued scams grazers. They determine their belonging to a category of cybercriminals while strengthening the perpetuation of scams on the web activities.
\end{abstract}

Keywords: "'grazer' 'Cybercrime - festive practices - Social Categorization..

\section{Résumé}

Le présent article vise à expliquer le mécanisme de catégorisation sociale qui régit l'univers des cybercriminels. C'est donc un examen des pratiques du «brouteur», notamment les pratiques festives (sexe, alcoolisme, psychotropes, etc.) que la démarche ethnographique a permis de faire. L'analyse des données tant qualitative que quantitative, mets en lumière la complexité du processus de typologisation sociale du cybercriminel ivoirien et des pratiques festives qui s'y rattachent. Ainsi le quotidien du "bouteur » et le caractère ritualiste des pratiques sociales existantes ont été observés sur une année (février 2012 - janvier 2013), auprès de 250 enquêtés dont 100 cybercriminels sur l'ensemble des 10 communes d'Abidjan. Cela a permis de se rendre à l'évidence que les 
pratiques festives sont les fins que poursuivent les arnaques des brouteurs. Elles déterminent leur appartenance à une catégorie de cybercriminels tout en renforçant la perpétuation des activités d’arnaques sur le web.

Mots clés : «’ Brouteur »’, Cybercriminalité — Pratiques festives — Catégorisation sociale.

\section{Introduction : quelques repères théoriques}

Le développement de l'Internet et sa croissance continue ont un impact significatif sur le développement des sociétés à travers le Monde. Cette évolution a permis de faciliter l'accès aux connaissances et a multiplié et accéléré la communication entre les personnes, sans tenir compte de la distance géographique. On ne saurait se priver de l'outil informatique aujourd'hui, en effet, au regard des nombreuses possibilités qu'il offre à ses millions d'utilisateurs situés à tous les coins de la planète et dans tous les secteurs d'activités. Néanmoins, le web 2.0 ou l'Internet facile d'utilisation a également provoqué une évolution vive de la cybercriminalité qui en Côte d'Ivoire est connue sous le vocable de «broutage ». Autrefois l'apanage de quelques spécialistes en informatique, le broutage a pris de l'ampleur, incluant des jeunes sans compétences pointues en informatique. Ainsi, tous constateront que la Côte d'Ivoire n'est pas restée en marge de ce phénomène qui fait l'objet de vives discussions et ne laisse aucun acteur social en marge du débat. Selon FREYSSINET (2012), les NTIC ne connaissent pas par nature de frontières physiques. Ils élargissent ainsi considérablement le périmètre d'action du cybercriminel. Il met donc l'accent sur les méthodes d’abus utilisées par les cybercriminels pour opérer. Il présente également un état des lieux quasi exhaustifs de la situation de la cybercriminalité tant au niveau technique que juridique, mais également au niveau des différents acteurs de ce «business » organisé. GAWA (2014) estime que la situation ivoirienne du «brouteur» correspond sur beaucoup de points à celle du « Feynman » camerounais dont la réussite repose sur l'arnaque des autres et se rapproche encore moins des descriptions faites par HAVARD sur les nouvelles figures de la réussite au Sénégal via le sport et la musique. Il évoque aussi qu’en Côte d’Ivoire, la danse du coupé décalé contribue consciemment ou inconsciemment à légitimer ces mécanismes illicites de l'enrichissement en louant davantage les mérites de ces nouveaux adeptes du « broutage » qui sont en passe de conquérir une place de poids sur l’échelle sociale du pouvoir. Toujours selon GAWA, comme le Feynman camerounais, le talent de ces jeunes réside dans l'arnaque consistant à faire croire à leurs victimes, généralement expatriées, qu’ils sont détenteurs de titres donnant l'occasion de faire des affaires privées et parfois douteuses dont l'objectif principal reste le détournement illicite de fonds. Comme 
l'expliquent BAZENGUISSA et GAFFEY (1995: 28), « cet imaginaire implique une dimension de banditisme où tout est permis et justifié par l'idée qu’il faut se débrouiller (...) c'est-à-dire un mode de récupération qui implique un mode de légitimité, un devoir impératif qui engage «non seulement l'individu, mais toute la communauté [...]», et rompre avec la dégradation progressive des conditions de vie dans les villes et le manque d'espoir pour une jeunesse dont les seuls horizons sont « les bidonvilles aux alentours des villes ultramodernes » (NZAU, 1984 : 21). Ce qui en fait au regard du contexte ivoirien des sujets « conscients d’être les acteurs malgré tout, d'une révolution qui ne dit pas son nom ». En somme selon (DJUNGUSIMBA 1996: 77), l'usage des moyens détournés à des fins d'enrichissement personnel via le réseau internet et d'autres mécanismes ont pour but le contournement du système légal national, mais aussi du système international. L’usage des moyens détournés prive toujours une plus grande partie des « gens sans part » et d'individus des dividendes politiques et du commerce des échanges qui se déroulent au niveau des scènes nationales. SIMBA présente donc le rôle du coupé décalé via la figure sociale du « brouteur ». Pour clore, il dira que ces différents airs et rythmes du coupé décalé s’emboitent dans les logiques de la globalisation et son message principal se retrouve dans une posture festive où elle offre l'occasion à ses différents adeptes de se divertir malgré la présence de la crise (qu'elle soit mondiale ou nationale). Aussi la vie moderne dans les grandes métropoles est largement rythmée par un ensemble d’institutions dont l'école, l'église, les associations culturelles, les partis politiques si bien que le temps des loisirs est utilisé pour se soustraire des obligations institutionnelles devenues assez contraignantes pour les individus et les communautés. (DJUNGUSIMBA 1996 : 77) fais une contribution explicative du succès actuel du coupé décalé notamment dans ses rapports étroits avec l’imaginaire politique des jeunes citadins (qui à travers ce rythme musical, élargissent les frontières sociales du pouvoir en Côte d'Ivoire ? Ces derniers, ainsi, remettent en crise l'efficacité symbolique du discours politique en le transformant comme un facteur dynamisant de repositionnement sur l'échelle sociale du pouvoir. Les recherches effectuées sur la cybercriminalité ont facilité une compréhension globale de cette notion, bien qu'il soit difficile de donner une définition uniforme de cette nouvelle forme de criminalité. Ces différentes études menées par des chercheurs ou organismes, ont fait une fixation sur les facteurs explicatifs de la cybercriminalité, ses manifestations, son évolution spatio - temporelle, ses impacts en termes de pertes financières et matérielles, les risques présents sur le cyberespace et les moyens de lutte contre le phénomène. Elles ont ignoré ou ont négligé (hors mis BAZENGUISSA et GAFFEY) un aspect essentiel de cette étude en l'occurrence : les rapports des cybercriminels avec les festivités. C'est cette 
étude qui nous intéresse en tant qu’objet dans cet article. Car à ce sujet, la fête est un phénomène social universel qui se croise dans toutes les cultures. Elle peut souligner les passages (saisons, la naissance, la mort, le changement de statut...). C'est donc un temps « à part », une ponctuation. Et surtout une rupture avec le temps ordinaire soumis à des règles générales : avec la fête ce sont les transgressions, la démesure, selon le colloque d’adrénaline (2015).

A la notion de fête donc, on accorde une dimension cérémonielle. Dans le contexte de la cybercriminalité pour rejoindre BAZENGUISSA et GAFFEY, même sous un volet sous culturel, la fête est une affirmation du brouteur, une mise en scène d'un ordre social, où des identités instituées sont hiérarchisées. C'est aussi une prégnance des codes, des règles, des rituels, du respect des conventions, «une étiquette ». La fête en définitive, est la célébration de l'ordre habituel accentuée par une mise en scène de soi et le respect des conventions sous-culturelles du brouteur.

La fête a aussi une dimension carnavalesque, où il s'agit d'inversion, d'un retournement des identités et des valeurs. C'est la recherche du vertige et de la démesure, une licence qui autorise ce qui n’est pas permis en temps normal. On fusionne avec l'autre pendant les festivités et on est indifférent à toutes les considérations. On perd aussi l'horizon temporel et c'est l'immédiateté du vécu qui est le plus important en ce temps « $\mathrm{T}$ ». La fête libère les émotions et pulsions fait installer un désordre provisoire qui vient consolider l'ordre habituel par contraste. Cependant, les évolutions conduisent à banaliser l'esprit festif, les animations festives permanentes. Là, la fête perd son caractère exceptionnel, qui est une fonction de passage limite fête/loisirs. Partiellement la cérémonie disparait avec moins de contraintes, d'obligations seulement une recherche du plaisir sans frein, de l'hédonisme. Cet effet, lorsque la fête chez les Brouteurs est en perte de vitesse, l'ivresse, la consommation de psychotropes entre en ligne de compte afin de mieux renforcer la recherche du plaisir de l'évasion tous azimuts. Cette situation met en évidence la précocité accrue des expérimentations de psychotropes en général et surtout de l'alcool en particulier sans doute même le sexe.

Ces Pratiques des Brouteurs sont moins socialisées par les adultes d'où le fait, de la stigmatisation des pratiques festives des jeunes brouteurs. De tout ce qui précède, l'on peut retenir un paradoxe: l'orientation permissive des relations familiales opposée à l'univers de la fête et aux conduites excessives cachées aux parents: attitudes et agissements des jeunes brouteurs vis-à-vis de leur famille. Cette étude vise à parvenir à une anthropologie de la fête en tant que manifestation dans la construction sociale du brouteur. L’étude codifie et surtout fait ressortir les transgressions qui y ont lieu. Les différents types de consommation des jeunes Brouteurs 
nous intéressent, de même que les rassemblements festifs. Toute cette stratégie aide à mettre en place les profils de fêtards des jeunes brouteurs : ce qui fait ressortir un modèle d'identification qui différencie le cybercriminel parmi d'autres jeunes du même âge qu'eux, qui eux ne sont pas cybercriminels.

Dans le présent article, l’étude tente de présenter que la recherche qualitative de type ethnographique constitue un des instruments des plus appropriés pour comprendre le processus de construction identitaire des jeunes Brouteurs ivoiriens à travers les différentes pratiques festives en ce qu'elles se prêtent à une analyse microsociologique.

\section{Méthodologie}

\section{- Délimitation spatio - temporelle de l'étude}

L'étude s'est effectuée sur l'ensemble des 10 communes de la ville d'Abidjan. En effet, dans chacune de ces communes, l'on note la présence ou l'existence de « brouteurs ».

L’enquête a été menée dans les maquis - bars des différents quartiers parce que ce sont les milieux qui sont réputés accueillir les festivités auxquelles prennent part les «brouteurs ».

Vu le nombre élevé de maquis — bar à Abidjan, nous avons choisi de visiter 3 maquis - bars (fréquentés par les «brouteurs ») par commune. Ainsi, le quotidien du «brouteur » et le caractère ritualiste des pratiques sociales existantes ont été observés sur une année (février 2012 — janvier 2013).

Tableau $n^{\circ} 1$ : Nombre de maquis et bars visités par commune.

\begin{tabular}{|c|c|c|}
\hline $\mathrm{n}^{\circ}$ & Communes & Maquis - bars visités \\
\hline 1 & Abobo & DOKOUI VIP - LEGENDE II - LE SPOT D’ABIDJAN \\
\hline 2 & Adjamé & ZENITH- MIDRUDE- MATION \\
\hline 3 & Attécoubé & LA TERRASSE - FASHION BAR - TOURE EIFFEL \\
\hline 4 & Cocody & VIP BAR - ESPACE OPERA - 11 D’AFRIQUE \\
\hline 5 & Koumassi & $5^{\text {eme }}$ DIMENSION - CHEZ ISY - 123 LOVE \\
\hline 6 & Marcory & FACEBOOK - MILLE MAQUIS - PRESIDENCE \\
\hline 7 & Plateau & LIFE STARS - ESPACE COCA COLA - VIP NIGHT CLUB \\
\hline 8 & Portbouet & ALBATROS - LA CUVETTE - ESPACE CHOCO \\
\hline 9 & Treichville & KAWAÎ- GARGAMEL- QUARTIER APOLO \\
\hline 10 & Yopougon & DIESEL - CYCLONE - BOUSING \\
\hline
\end{tabular}

Source : Notre enquête.

\section{- Échantillon}

100 cybercriminels ont participé à l’enquête à travers le récit de leurs expériences festives, en raison de 10 «brouteurs » par communes.

Le choix des enquêtés dans chacune des communes s’est fait de façon non probabiliste. 
En plus des brouteurs, 50 clients de maquis et bars, 30 gérants de maquis et bars, 70 spectateurs des festivités dans les maquis et bars ont été interrogés. Soit un total de 250 enquêtés.

\section{- Instruments de recueil des données}

Les données ont été recueillies à l'aide de la recherche documentaire, l'observation directe et l'enquête - interrogation.

En effet, une documentation sur la cybercriminalité en général et les acteurs impliqués dans ce phénomène a été consultée.

Dans les maquis visités, nous avons pu observer les « brouteurs » lors de leurs différentes prestations (« travaillement », offre gratuite de boisson à tous les clients, « le boucan », etc.).

Enfin, l'enquête - interrogation a permis d'interviewer les enquêtés sur leur connaissance de la cybercriminalité et des buts et finalité des pratiques festives des « brouteurs".

Mais quelle stratégie avons-nous adoptée pour approcher notre population cible? En fait, nous avons privilégié l'enquête ethnographique. En effet, l'ethnographie qui consiste à être dans le quotidien et les habitudes des brouteurs, nous a aidé à cerner les tenants et aboutissants des pratiques festives des brouteurs. Loin d'être un phénomène normalement passager dans les habitudes ordinaires, la fête est démesurée dans le quotidien des brouteurs. Ainsi, pour faciliter le rapprochement, il fallu gagner la confiance des enquêtés. Nous avons présenté aux brouteurs « leur finalité » qui consiste à aider les délinquants à mieux faire connaitre leur cri de cœur aux adultes. Rassurer par la justesse de la finalité de l'étude, les participants ont adhéré à la recherche en se laissant approcher.

\section{- Méthodes d'analyse}

Les données ont été analysées tant qualitativement que quantitativement.

L'analyse qualitative a aidé à la mise en place d'une typologie du brouteur ivoirien relativement à son état mental ou émotionnel face aux pratiques festives qui apparaissent déterminantes dans les fins poursuivies par les activités d'arnaqueurs.

L'analyse quantitative montre le taux et le niveau d'addiction des cybercriminels dans les pratiques festives au niveau de la consommation de substances nuisibles à leur équilibre mental et moral.

Résultats :

Pratiques festives des brouteurs : typologie et ampleur

Typologie des pratiques festives des brouteurs en fonction de l'âge et du sexe 
Tableau 2 : Pratiques festives du Brouteur (les consommations) par tranche d'âge et sexe

\begin{tabular}{|c|c|c|c|c|}
\hline $\begin{array}{c}\text { Pratiques festives } \\
\begin{array}{c}\text { Enquêtés } \\
\text { par tranche d'âges/sexe }\end{array}\end{array}$ & $\begin{array}{c}\text { Consommation } \\
\text { alcool }\end{array}$ & $\begin{array}{c}\text { Consommation } \\
\text { sexe }\end{array}$ & $\begin{array}{c}\text { Consommation } \\
\text { Psychotropes ou } \\
\text { stimulants }\end{array}$ & Effectifs \\
\hline $14-16$ ans masculins & 6 & 2 & 1 & 9 \\
\hline $14-16$ ans féminins & 1 & 2 & 0 & 3 \\
\hline $16-18$ ans masculins & 10 & 6 & 1 & 6 \\
\hline $16-18$ ans féminins & 3 & 2 & 3 & 25 \\
\hline 18ans-20ans masculins & 13 & 9 & 1 & 10 \\
\hline 18 ans-20 ans féminins & 5 & 4 & 5 & 18 \\
\hline+25 ans masculins & 8 & 5 & 2 & 10 \\
\hline+25 ans féminins & 4 & 4 & 16 & 100 \\
\hline Effectifs & 50 & 34 & & \\
\hline
\end{tabular}

Source : l'enquête

Commentaire: sur une population de sexe masculin de 71 participants, 37 reconnaissent consommer de l'alcool. Sur les 29 de sexe féminin, 13 reconnaissent consommer de l'alcool. Au total 50 participants reconnaissent consommer de l'alcool. Sur les 71 masculins 22 reconnaissent consommer du sexe. 12 de sexe féminin reconnaissent consommer du sexe sur les 29 de la population échantillon soit un total de 34. Sur les 71 masculins de l'échantillon14 participants Consomment les Psychotropes ou stimulants. Sur les 29 sexes féminins 4 Consomment les Psychotropes ou stimulants soit 16 au total.

\section{Ampleur et fréquence des pratiques festives des brouteurs}

Tableau 3 : Taux de pratiques festives des brouteurs par tranche d'âges/sexe

\begin{tabular}{|c|c|c|c|c|}
\hline $\begin{array}{c}\text { Enquêtés par tranche } \\
\text { d’âges/sexe }\end{array}$ & $\begin{array}{c}\text { Pratiques } \\
\text { festives faibles }\end{array}$ & $\begin{array}{c}\text { Pratiques } \\
\text { festives } \\
\text { moyennes }\end{array}$ & $\begin{array}{c}\text { Pratiques } \\
\text { festives élevées }\end{array}$ & Effectifs \\
\hline 14-16ans masculins & 5 & 2 & 2 & 9 \\
\hline 14-16 ans féminins & 1 & 2 & 0 & 3 \\
\hline 16-18ans masculins & 4 & 6 & 9 & 19 \\
\hline 16-18 ans féminins & 1 & 2 & 3 & 6 \\
\hline 18-20ans masculins & 1 & 5 & 19 & 25 \\
\hline 18-20 féminins & 1 & 4 & 5 & 10 \\
\hline +25 masculins & 2 & 5 & 11 & 18 \\
\hline +25ans féminins & 2 & 2 & 6 & 10 \\
\hline Effectifs & 17 & 28 & 55 & 100 \\
\hline
\end{tabular}

Source : l'enquête

Commentaire: sur une population de sexe masculin de 71 participants (12 reconnaissent avoir des Pratiques festives faibles. Sur les 29 de sexe féminin ( 7 reconnaissent avoir des Pratiques festives faibles. Au 
total 17 participants reconnaissent avoir des Pratiques festives faibles. Sur les 71 masculins 18 reconnaissent avoir des Pratiques festives moyennes. 10 de sexe féminin reconnaissent avoir des Pratiques festives moyennes sur les 29 de la population échantillon soit un total de 28. Sur les 71 masculins de l'échantillon 41 participants reconnaissent avoir des Pratiques élevées. Sur les 29 sexes féminins 14 reconnaissent des Pratiques élevées soit 55 au total. Tableau 4 : Fréquence des pratiques festives des brouteurs au niveau de l'ivresse, selon l'âge

\begin{tabular}{|c|c|c|c|c|}
\hline $\begin{array}{c}\text { Enquêtés par tranche } \\
\text { d'âges/sexe }\end{array}$ & $\begin{array}{c}\text { Ivresses au } \\
\text { moins } 1 \text { fois }\end{array}$ & $\begin{array}{c}\text { Ivresses au } \\
\text { moins } 2 \text { fois }\end{array}$ & $\begin{array}{c}\text { Ivresses au } \\
\text { moins } 3 \text { fois }\end{array}$ & Effectifs \\
\hline $14-16$ ans masculins & 6 & 1 & 2 & 9 \\
\hline $14-16$ ans féminins & 2 & 1 & 0 & 3 \\
\hline 16-18ans masculins & 10 & 4 & 5 & 19 \\
\hline $16-18$ ans féminins & 3 & 2 & 1 & 6 \\
\hline $18-20$ ans masculins & 10 & 6 & 9 & 10 \\
\hline $18-20$ ans féminins & 4 & 3 & 3 & 18 \\
\hline+25 ans masculins & 7 & 3 & 8 & 10 \\
\hline +25 ans féminins & 5 & 3 & 2 & 100 \\
\hline Effectifs & 47 & 23 & 30 & \\
\hline
\end{tabular}

Source : l'enquête

Commentaire: sur une population de sexe masculin de 71 participants $6+10+10+7$ (33 reconnaissent avoir une fois au moins été ivres lors des parties festives. Sur les 29 de sexe féminin 2+3+4+5 (14 reconnaissent avoir une fois au moins été ivres lors des parties festives. Au total 47 participants reconnaissent avoir une fois au moins été ivres lors des parties festives. Sur les 71 masculins 14 reconnaissent avoir deux fois au moins été ivres lors des parties festives. 9 de sexe féminin reconnaissent avoir deux fois au moins été ivres lors des parties festives sur les 29 de la population échantillon soit un total de 23. Sur les 71 masculins de l'échantillon de 24 participants reconnaissent avoir trois fois au moins été ivres lors des parties festives. Sur les 29 sexes féminins 6 reconnaissent avoir trois fois au moins été ivres lors des parties festives soit 30 au total

quand est-il de la Typologie des comportements festifs du brouteur ivoirien?

\section{Comportements festifs et profil du cybercriminel ivoirien}

L’étude se réfère par analogie aux enquêtes IREB (2009 et 2010). Elle est parvenue à faire une typologie du cybercriminel brouteur ivoirien. Comportements qui vont se différencier en fonction de l'histoire, de la construction identitaire; des valeurs, de la vision de l'avenir... Ainsi, certains brouteurs font du «sur place » pendant que d'autres avancent. On parlera de ce fait d'une part d' «habilité sociale » et d'autre part de « régulation émotionnelle » pour qualifier ces comportements festifs. 
Tous fêtards, les brouteurs font l'alternance de la recherche de la liberté et de la recherche de l'autonomie. Ils recherchent une alternance idéale à travers la fête qui devient synonyme d’ivresse de joie démesurée, de gaspillage d'argent entre copains. Et cette façon de faire devient de la sociabilité culturelle. En d'autres termes, notre regard de criminologue, nous amène à parler de sous culture résiduaire où l'alcool, le vice et bien d'autres stimulants pour vivifier la fête deviennent l'apanage de ces jeunes brouteurs. Toujours à la recherche d'une alternance idéale, le jeune brouteur arrive à alterner le temps de l'école, des études et les temps festifs. Il parvient malgré les risques liés à son métier à se projeter dans le temps. Les lieux de prédilection des brouteurs fêtards commencent par des appartements d'amis, où on organise des fêtes initiatiques. Puis vient le temps ou l'épisode des discothèques tous les soirs. Lorsqu'il arrive que le brouteur acteur de joie et de fête arrive à se sentir mal à l'aise, il se produit une sorte de précarité du lien social et l'individu se mue dans une ivresse angoissante.

\section{Le brouteur « mal à l'aise "}

L'étude nomme cet individu "le mal à l'aise ", c'est un brouteur à sociabilité forte et restrictive. Il a un regard critique sur les comportements festifs liés à l'ivresse, à la démesure et à la joie tous azimuts.

Il est à la fois attiré et repoussé par les fêtes. Son encadrement familial semble plus fort par rapport à d'autres types de cybercriminels.

Voici illustré les paroles d'un « mal à l'aise » interrogé au Maquis V 12 à Adjamé (Renault) :

« Je ne suis pas du tout dans ce truc là, les bistrots je ne m’y sens pas forcement bien ».

Un autre rencontré au VIP BAR de Cocody affirme : "La fête, je la vois en spectateur. Ma communion avec les autres, elle ne se passe pas là dedans. Parce qu'il n'y a pas de liens de confiance, je me sens complètement en dehors »

$\mathrm{Au}$ maquis - bars "CHEZ ISY " à KOUMASSI, Mathieu BECEAO (un mal à l'aise) dira à propos des maquis bars : "L'appartement, ce n'est pas un terrain neutre, c'est un lieu qui n'est pas public. T'es dans le territoire de quelqu'un. Y a quelque chose comme de l'admission : tu rentres sur son terrain, tu rentres chez lui, tu vis avec ton pair quoi ».

\section{Le brouteur " conformiste "}

Le Brouteur "conformiste », par contre, a des relations plus fortes avec la société des brouteurs. Il valorise peu la jouissance; il a une sociabilité forte, une tendance habituelle à la restriction. Le brouteur conformiste entre dans la fête par mimétisme au collectif des copains. 
L’alcool, l'ivresse, la fête et l'amusement sont pour lui une sorte d'atteinte d'une régulation par le monde des adultes.

Illustration à travers les paroles de brouteurs « conformistes ».

MIKE LA DOUCHE (un conformiste, Maquis ALBATROS PORTBOUET) : "Se retrouver dans un cadre nouveau te fait dire des trucs que tu ne dirais pas normalement. Qu'il y ait une telle ébullition autour de la préparation d'une fête c'est bien qu'on sent que les rapports vont changer ».

TEGUE LE PIGEON VERT (Conformiste, Resto bar TOURE EIFFEL, ATTECOUBE) : «Il y avait quand même un programme à respecter plus ou moins. C'était en début de soirée chez un particulier qui finit dans un club. »

JOJO TEMOIN (Conformiste, Maquis bars LA

TERRASSE, ATTECOUBE quartier Ebrié): "J'essaie de bien me coiffer, bien m'habiller, touche de parfum, avoir un paraître irréprochable. Je pense que je me prépare comme toute personne normalement constituée. J'essaie de faire tout pour plaire un minimum aux personnes qui vont m'entourer et que je vais côtoyer tout au long de la soirée ».

ABOBOLAIS LA COMPAGNIE (Conformiste, Bars CYCLONE, YOPOUGON) : "Quand ça fait une bonne partie, un bon laps de temps que tu côtoies les mêmes personnes en soirée, tu sais comment ils réagissent et puis tu commences à connaître toutes tes limite. Donc après un certains âge tu dis que c'est bidon d'aller jusqu'à se rendre malade, perdre totalement le contrôle de soi ».

\section{Le brouteur « libertin »}

Le brouteur « libertin » a une relation plus forte avec le groupe de pair, il aime les jouissances fortes et il fête pour délirer.

Le «libertin» connait une sociabilité forte avec une jouissance effrénée. Il valorise la fête et aime appartenir à une communauté émotionnelle. Le "libertin » partage une esthétique commune, il aime les sorties précoces qui se prolongent dans le cycle de la vie (même après installation en couple). Les drogues stimulants (alcools forts, la coke, les ecstasys) justifient idéologiquement les comportements de ce type de brouteur.

La recherche d'authenticité pousse le brouteur « libertin » à vouloir toujours profiter de sa jeunesse, dans son cycle de vie segmentée. Le « libertin » renouvelle constamment les réseaux de sociabilités. Les pairs jouent un rôle important pour son «auto surveillance». Pour lui, la responsabilité collective est un idéale. Il se mue dans un sentiment illusoire de sécurité. 


\section{Illustration à travers les paroles du brouteur libertin}

GEORGINIO LE MILLIARDAIRE (Maquis DOKOUI VIP, $\mathrm{ABOBO})$ : "On se retrouve des fois à 30 ou 40 dans un appartement et j'en connais que dix ou quinze. Tout le reste, je les connais pas (...) moi je rencontre des gens que je ne connais pas, et ils me disent "ah, mais moi je suis venu parce que je connais un tel et qu'il m'a invité". c'est comme ça que se rencontrent les gens, et puis que les réseaux se forment. Après on les invite à d'autres soirées ».

MANU LA TASSE DE PIPI (Maquis bars GARGAMEL, TREICHVILLE) : "J'ai plus besoin de voir mes amis que mes parents. C'est vraiment fort entre nous avec mes groupes de pairs ».

TINO LE MOBILISATEUR MOBILE (Maquis bars KAWAÏ, TREICHVILLE) : "On est au dessus de la moyenne. Je compare par rapport aux gens qui sont dans ma catégorie ou d'autres gens. C'est sûr, y en - a qui se mettront jamais minables comme moi... mais bon ; j'aime ce style de vie. ».

TINTIN LA VIVACHET (MILLE MAQUIS, MARCORY) : "Mes parents boivent beaucoup moins que moi. Ils ne savent même pas la vérité sur ce que je bois parce que... ils peuvent pas imaginer que je me bois tout seul un pack de 24 bières en une soirée (...) on sait la limite, mais pourtant on a beau avoir 22 ans, des fois on la dépasse ».

\section{Le brouteur « casse cou »}

Le " casse cou » ou fuite de soi est incapable de s'arrêter. Il est toujours en fête pour s'absenter des réalités de la vie. Le brouteur casse cou vit dans un malaise existentiel ; il cherche à fuir la réalité.

Il ne parvient pas à se maîtriser face à tout genre de consommations, qu'elles soient chroniques ou isolées. Ils a également recours aux drogues anesthésiantes « kétamine, à l'alcool à forte dose et quelque fois à des doses de cocaïne et d'héroïne. Le brouteur casse cou est plutôt un garçon, qui aime s'isoler de ses pairs différemment du brouteur conformiste qui recherche son équilibre à travers les fête de groupe et ses pairs.

Le brouteur " casse cou » vit dans une instabilité relationnelle raison pour laquelle il a tendance à vivre dans l'isolement. Il s'installe dans une indépendance avec rupture à l'égard des parents ou de l'autorité supérieure.

Les brouteurs en général, aiment les sorties et surtout les expérimentations précoces dans le cycle de la vie (l'école) et en progression jusqu'à tard dans l'âge de jeune adulte. A les écouter, ils reportent l'installation en couple. C'est une période discothèque intensive puis avec des diversités de soirées dans les bars, chez des amis, à des concerts, à des festivals, "l'occasion fait le larron ». Leur caractéristique générale, c'est le manque de cohérence chronologique entre le temps des fêtes et le temps 
habituel. Le brouteur dénie l’accident ; il a une prégnance du risque. Il réagit certes au drame ou à la répression, mais pas dans la durée. Il se met en danger en cas de rupture avec son mode de vie qui consiste à être toujours en soirée (partir seul, « Peter un plomb », décompensation psychiatrique).

Illustrations à travers des paroles du « casse cou »

HIGHFINGER LE RICHARD INTOUCHABLE, ESPACE OPERA, COCODY) : « Faire des mélanges (d'alcools forts), c'est nocif, mais j'aime bien ça».

RODJA L'INTARRISSABLE, Maquis BOUSING, YOPOUGON Figayo) : " Je ne me suis jamais bien entendu avec mes parents. Quand je ne les vois pas, ça va bien. Si je les vois trop souvent, ça devient une prise de tête autour de la fête, de la défonce ».

Ainsi, nous distinguons quatre (4) types de brouteurs: le « conformiste ", le " libertin ", " le mal à l'aise " et " le casse cou ".

Tout un chacun a une caractéristique propre vis-à-vis des pratiques festives : le « conformiste » fête pour se sentir intégré dans l'assemblée et par les pairs. Le « libertin » quant à lui, fête pour délirer. Le «mal à l'aise » fête, pour se mettre à distance des réalités de la vie.

Ce qu'ils ont en commun, c'est leur âge, leur degré de prendre des risques et surtout, ils sont de grands fêtards (Cet Ivoirien adepte du broutage dépense le gain de ses larcins en hôtels, filles et fringues. Sans scrupules, il se rêve en Robin des Bois). D'aucuns se repli sur eux-mêmes avec grande limitation dans les relations avec les pairs, d'autres fusionnent facilement avec les autres pairs. À travers les différentes paroles ces jeunes brouteurs montrent qu'ils sont fortement proche de l'alcool, des psychotropes et drogues pendant les instants festifs. D’une part pour la mise en œuvre de leur mode opérateur, ils vont s'installer dans une criminalité organisée basée sur des réseaux ou des complicités. Dès que le gain est perçu, les brouteurs vont s'installer dans une sous - culture résiduaire caractérisée par les fêtes, les jouissances; les amusements. On commence par fêter un événement quelconque dans l'appartement d'un tiers pair. Par la suite, on s'invite dans les discothèques où on fait "couler l'alcool à flot", on gaspille les gains obtenus par arnaque sur le web. On invite les pairs à des fêtes où tous les coups sont permis drogues psychotropes, hallucinogènes, sexe. Très souvent jeunes et adolescents, ils ont un âge qui tourne autour de 14 ans ou même 20 ans et plus. Le plus souvent, ils appartiennent à toutes les classes sociales ivoiriennes, puisque les cyberespaces sont démocratisés. En Côte d'Ivoire, à tout coin de rue, on peut trouver un cybercafé. Ainsi, aucune des dix communes qui constituent la ville d'Abidjan n'est épargnée par ce type d'activité. Les jeunes filles rares au départ du phénomène sont de plus en plus présentent dans le réseau et participent aux pratiques festives comme les jeunes garçons adolescents (29 participantes). 


\section{Discussion}

Des auteurs comme Nahoum Grappe, affirme que la fête est de la ritualisation, du cérémonial ; c'est-à-dire que l'entrée en fête donne un sens aux différentes transgressions présentées ci-dessus. La reflexité concerne : photos, souvenirs, anecdotes liées à la fête. Le genre et la répartition sexuée des tâches (rôle protecteur des filles, manger, dormir..., modèles identitaires) sont opposés ici. Ce sont: la virilité contre le maternage. La drogue et l'ivresse excessive contre les relations amoureuses. Ces antinomies donnent un contenu, un sens à la fête dans laquelle demeure généralement les jeunes brouteurs. L'inter - génération est une sorte de prise de distance avec soi pour une diffusion culturelle. La scène, la dance, le « live »... donnent du contenu à la fête contre le vide, l'ennui. Autrement dit, les brouteurs créent la fête pour fuir l'ennui, le vide. Dans les travaux de Freyssinet Dominjon et Wagner (2004), on se rend compte que l'alcool devient un code de consommation pour les jeunes adolescents en général et chez les jeunes brouteurs ivoiriens en particulier; c'est un style d'affirmation du vécu. Le jeune brouteur à travers ce mode de consommation et le temps de fête qui s'associe, se forge une identité au-delà de son aspect transgressif pour ce qui concerne le regard des adultes. Les résultats collectés au tableau 2 montrent que l'âge des participants varie entre 14 ans et 25 ans justifiant l'hypothèse selon laquelle nous avons en présence des participants dans la fleur de l'âge. Au cours des pratiques festives les cybercriminels confirment avoir touché au moins une fois à de l'alcool, aux psychotropes ou tous autres stimulants et avoir pratiqué du sexe. Et ces données sont justifiées par les recherches de (Cohen, 1955) qui à aborder la question de sous-cultures criminelles. On peut dire que les participants rentrent jeunes dans la délinquance virtuelle on peut parler de sous-culture de communauté virtuelle selon (Wellman et Gulia, 1999). Les participants passent par l'arnaque pour obtenir des gains qui leur servent à enjoliver les séances ou pratiques festives qui sont le couronnement de leur activité sur le web. Au cours des pratiques festives, l'alcool, les psychotropes ou le sexe sont usités à fond. Selon (Monique Dagnaud, 2008) toutes ces pratiques sont liées à une recherche d'identification ou d'opposition des jeunes au monde des adultes. Selon Cohen dans une vie sous culturelle les jeunes s'associent pour se braquer contre la société des adultes qui est trop normative. La vie dans les bandes délinquantes à ses propres codes qui s'opposent à celle du monde conventionnel d'où la démesure observée pendant les pratiques festives du «Brouteur ». Et les pratiques festives permettent à l'étude d'établir une typologie des participants. Ainsi la collecte des données a permis de révéler des cybercriminels qui font dans l'excès, d'autre dans la neutralité et certains dans un pseudo conformisme. Les études de (Martin Bouchard, et Bryce Westlake, 2016) démontrent combien de fois les «brouteurs" même 
embarqués dans une Co-délinquance due à leur implication dans un réseau d'activités criminelles ont des particularités. Et cette approche justifie aisément la typologie retracée ci-dessus dans la collecte des données. (McGloin et Nguyen et al, 2013) disent que la sélection des Co délinquants ne se fait évidemment pas de manière aléatoire. Les délinquants regardent d'abord dans leur propre environnement social pour trouver leurs complices. Selon (tremblay, 1993 ; Morselli et Tremblay, 2004 ; Nguyen et Bouchard 2013), les plus aguerris prendront un peu plus de risques en diversifiant leurs sources de capital social criminel pour ainsi en tirer des bénéfices additionnels non accessibles autrement. Bien que les tendances générales en co-délinquance soient relativement bien connues, plusieurs aspects de la coopération criminelle font encore l'objet de trop peu de recherches empiriques. Les implications pour la co-délinquance de l'utilisation sans cesse grandissante de l'informatique et d'internet pour faciliter la perpétration de crimes est l'un de ceux-là. Peu d'auteurs ont en outre amorcé le travail conceptuel qui consiste à évaluer si les différences entre la codélinquance en ligne et hors ligne nécessitent qu'on repense notre manière de catégoriser les relations entre codélinquants et la façon de les aborder en recherche.

\section{Conclusion}

Cette recherche s'est intéressée, en réalité, à la finalité d'une arnaque réussie du brouteur qui se matérialise par la fête. Les fruits du vol via internet partent, en effet, en direction de parties festives entre pairs. Et, quelles que soient les 10 communes d'Abidjan visitées, le constat montre les mêmes caractéristiques partout. Ainsi les 100 participants («brouteurs ») sont dans la majorité de grands fêtards. L’on retiendra ici que chaque brouteur présente une idée de fête. Pour certains c'est l'excès dans la transgression. Pour d'autres, ils y font avec un peu de retenu. Toutefois, l'enquête a pu montrer que la majorité des brouteurs présente un élan à la démesure, à la transgression dans les habitudes festives.

\section{References:}

BANEGAS, R. \& WARNIER, J.-P. (dir) (2001). Figures de la réussite et imaginaires politiques. Politique Africaine, $n^{\circ} 82$, Paris : Karthala.

BLE, R. G. (2006). Zouglou et réalités sociales des jeunes en Côte d'Ivoire. Afrique et développement, Vol XXXI N I, Dakar : CODESRIA, 168-184.

BAZENGUISSA, R. \& GAFFEY, M. (1995). Vivre et briller. Paris. Des jeunes congolais et Zaïrois en marge de la légalité économique. Politique Africaine, $\mathrm{n}^{\circ}$ 57, $124-133$

BOGUI, J.J. (2010). La cybercriminalité, menace pour le développement. Afrique contemporaine, Université du Qébec Montréal : PUM. 
COHEN, 1955, la culture des gangs, Free Press, United States of America COLLOQUE D'adrénaline, (2015). Rennes.

DEDJE, M. C. (2012). «’ Les brouteurs »’ défient le gouvernement ivoirien - Linfodrome

IREB (2009). Les facteurs de régulation des consommations : la fête en appartement ». Revue Place Publique, Rennes.

IREB (2010). Rôles professionnels de la nuit: Le cycle de vie (« installation » vers 20 et plus). Paris : éditions Jean-Pierre Gyss.

JORON, Ph. (2012). La fête à plein bords-CNRS, Paris

FREYSSINET, D. J. \& Wagner, A. C. (2004). L'alcool en fête : manières de boire de la nouvelle jeunesse, Paris : L'Harmattan.

GAWA, F. (2014). Le coupé décalé en Côte d'Ivoire : Sens et enjeux d'un succès musical. African Sociological Review, Vol 18 (1) ; 112- 126

GERIN, L. D. (2006). L'utilisation de l'ethnographie dans l'analyse des rapports à l'identité, éditeur de Boeck supérieur

KOLHLHAGEN, D. (2005). Frime, escroquerie et cosmopolitisme; Le succès du coupé décalé en Afrique et ailleurs. Politique Africaine, vol. 4 $\mathrm{n}^{\circ} 100,92-105$.

KOUAKOU, K. C. (2009). Jeunes et pauvreté urbaine dans les pays en développement : cas de la Côte d’Ivoire, Revue de l'intégration, Vol 3, $\mathrm{n}^{\circ} 1$, 1-34.

LA DEPECHE D’Abidjan (15 Février 2013). « Cybercriminalité - Côte d'Ivoire : 24 heures dans la vie d'un brouteur »

MARTIN Bouchard, et BRYCE Westlake, 2016 les réseaux criminels : p199, PUM

McGloin et Nguyen et al, 2013, les réseaux criminels : p199, PUM

NAHOUM Grappe, v. (2006). L’ennui est inséparable des moments festifs

- libération. Revue de Presse Internationale

NAHOUM Grappe, v. (1997). Les jeunes et la fête. Réseau Prisme. CNRSuniversité René Descartes Paris V, Paris

MONIQUE Dagnaud, 2008, la teuf, essai sur le désordre des générations, Seuil

TREMBLAY, 1993 ; MORSELLI et Tremblay, 2004 ; Nguyen et Bouchard 2013, les réseaux criminels : p199, PUM

WELLMAN et GULIA, 1999, les réseaux criminels : p199, PUM 